\title{
Landyne 4 - A Software Suite for Electron Diffraction Simulation and Analysis
}

Xing-Zhong Li

University of Nebraska, Lincoln, Nebraska, United States

Since Landyne 2 [1] was published in 2016, the software suite has been greatly upgraded. The current version includes ten stand-alone software components. Each of them was designed for one topic of application in simulation, analysis or data processing. A launcher is available for the software suite, which is a tool for the user to conveniently access all of the software components.

The purpose of this software suite is twofold: i) as practical tools in research work, ii) as teaching aids on microscopy and crystallography. The executable codes, user manuals and a set of crystal structural data are available at author's webpage, https://www.unl.edu/ncmn-enif/xzli/computer-programs. In this paper, the purposes and features of this software suite are briefly introduced. More details can be found in user manuals.

SVAT 4 [2] provides a 3D visualization of crystal structures, including chemical bonds and magnetic moments. Crystal structures can be analyzed (i) as a projection in a user-selected [uvw] direction or layerby-layer, (ii) as a local structure (or polyhedral cluster) by defining a center atom and a radius of spherical range. Figure 1(a) shows a structure visualization by using SVAT 4.

SAED 4 [3] is used for simulation of electron diffraction patterns on a single phase, twining and coexisted multiple phases with a fixed orientation and for determination of the zone axis from the experimental diffraction pattern. SAED 4 has an extension for projected atomic potential map. Figure 1(b) shows a simulated selected-area electron diffraction pattern by using SAED 4.

PCED 4, as an updated version of PCED2 [4], is for simulation of electron diffraction patterns from polycrystalline phase and for phase identification. The features include (i) an integral two-beam dynamical theory for intensity calculation, (ii) out-of-plane and in-plane texture and (iii) peak profile of diffraction ring.

SAKI 4 is used for simulation and analysis of Kikuchi patterns and also for simulation of double diffraction effect. A function is added on recently for the precise determination of crystal orientation using an electron diffraction pattern with three Kikuchi pairs [5].

SPICA 3 [6] is for stereographic projection with an application for specimen orientation adjustment using TEM holders. SPICA inherits JECP/SP functions and expands many new functions, e.g. Kikuchi map, for crystallographic analysis with a more user-friendly GUI design.

HOLZ 3, as an upgraded version of JECP/HOLZ [7], is an interactive program for simulation of the higher-order Laue zone (HOLZ) lines using kinematical approximation and a first-order dynamic correction. An improvement is for easy adjustments and a new display system to better show the labels for CBED and HOLZ patterns.

QSAED 3, as an updated version of JECP/QSAED [8], is used to quantitative retrieve/display the intensities of reflections on electron diffraction patterns and to measure line profiles on electron diffraction pattern. QSAED 3 has an extension for projected atomic potential difference map.

QPCED 3, as an updated version of QPCED 2 [9], is for digitization and quantification of polycrystalline electron diffraction patterns. It can also be used for correction of pattern with elliptical rings and display enhancement for a pattern with weak diffraction rings. 
TEMUC 3 [10] is a program to determine unit cell of crystalline phase in TEM using boththe reciprocal unit cell reconstruction approach and the reduced cell approach. Three approaches were implemented, including a traditional method from a tilt series of electron diffraction patterns and two reduced cell methods.

PAPM is an extension of SAED 4 and QSAED 3, for generating projected atomic potential (difference) map. It can be used to correlate to HR-STEM and HREM structural images and to improve structural model. Figure 1(c) shows a projected atom potential map by using PAPM. Figure 1(c) shows a projected atom potential map by using PAPM.
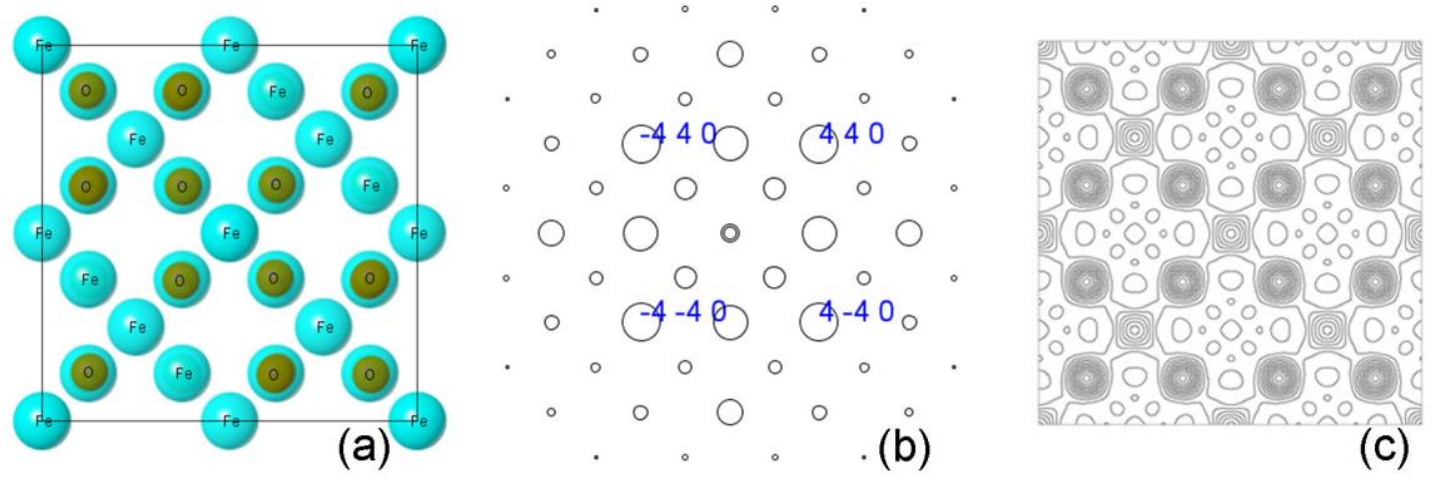

(c)

Figure 1. (a) A [001] projection of the Fe3O4 structure by using SVAT 4, (b) simulation of selected-area electron diffraction by using SAED 4 and (c) projected atom potential map by using PAPM.

\section{References}

[1] X.Z. Li. Microsc. Microanal. 22S3 (2016) 564-565.

[2] X.-Z. Li, J. Appl. Cryst. (2020) to be published.

[3] X.-Z. Li, Microsc. \& Anal. May issue (2019) 16-19.

[4] X.-Z. Li, Ultramicroscopy 110 (2010) 297-304.

[5] X.-Z. Li, J. Mater. Edu. (2019) submitted.

[6] X.-Z. Li, J. Appl. Cryst. 49 (2016) 1818-1826.

[7] X.-Z. Li, J. Appl. Cryst. 38 (2005) 576.

[8] X.-Z. Li, Microsc. Microanal. 20 (2014) 1486-1487.

[9] X.-Z. Li, J. Appl. Cryst. 45 (2012) 862-868.

[10] X.-Z. Li, X.Z. Li. Micron 117 (2019) 1-7. 